\title{
A clinical study on acupuncture in combination with routine rehabilitation therapy for early pain recovery of post-stroke shoulder-hand syndrome
}

\author{
JINLING ZHENG ${ }^{1}$, QINGLIAN WU ${ }^{1}$, LU WANG $^{2}$ and TING GUO ${ }^{3}$ \\ ${ }^{1}$ Department of Traditional Chinese Medical Rehabilitation, The Second Hospital of Dalian Medical University, \\ Dalian, Liaoning 116000; ${ }^{2}$ Department of Nursing, The Second Hospital of Dalian Medical University, Dalian, \\ Liaoning 116031; ${ }^{3}$ Cancer Clinic, The Second Hospital of Dalian Medical University, Dalian, Liaoning 116000, P.R. China
}

Received November 16, 2017; Accepted December 12, 2017

DOI: $10.3892 /$ etm.2017.5659

\begin{abstract}
The clinical effect of acupuncture in combination with rehabilitation therapy for post-stroke shoulder-hand syndrome (SHS) was explored. Patients (178) with post-stroke SHS who received treatment in the Dalian Second Hospital from March 2012 to March 2016 were included in this study. The patients were divided into experimental group (89 cases) and control group (89 cases). Patients in the control group received rehabilitation therapy, while those in the treatment group received acupuncture treatment in addition to rehabilitation therapy. Visual analogue scale (VAS) was applied to assess the pain degree of patients. Fugl-Meyer assessment (FMA), functional comprehensive assessment (FCA) and assessment of quality of life (QoL) were used to evaluate rehabilitation condition of the patients. Early pain relief, rehabilitation of upper extremity motor function and improvement of QoL after treatment were compared between the two groups. The scores of VAS, FMA, FCA and QoL showed obvious differences between the two groups after treatment $(\mathrm{P}<0.05)$. The scores of the experimental group were significantly better than those of the control group, and the improvement in upper extremity motor function of the patients in the experimental group was better than that of the patients in the control group. The total effective rate of the patients in the experimental group was higher than that of control group $(\mathrm{P}<0.05)$. The effect in improving the upper extremity motor function of the patients in the experimental group was better than that of control group. The scores of QoL of the patients in the experimental group were better than that of the patients in the control group $(\mathrm{P}<0.05)$. In conclusion, acupuncture in combination with
\end{abstract}

Correspondence to: Dr Ting Guo, Cancer Clinic, The Second Hospital of Dalian Medical University, 467 Zhongshan Road, Shahekou, Dalian, Liaoning 116000, P.R. China

E-mail: guoting2170@163.com

Key words: stroke, shoulder-hand syndrome, acupuncture, rehabilitation therapy, early pain recovery rehabilitation therapy can improve early pain and rehabilitation significantly and enhance QoL for patients with post-stroke SHS, which is worthy of being widely used in clinical practice.

\section{Introduction}

Shoulder-hand syndrome (SHS), also known as reflex sympathetic dystrophy (RSD) $(1,2)$, is one of the common complications after stroke. It occurs most frequently in the first two months after stroke and its incidence is $~ 70 \%$ (3). The main clinical symptoms include hemiplegic shoulder pain, hand edema and pain while moving. Symptoms such as muscle atrophy, tendon contracture and joint deformity on the affected side may be observed in some patients at the late stage of the disease. Severe cases may suffer from permanent deformity of the shoulder and the fingers, which will result in severe limitation in physical activity and great reduction in quality of life (QoL) of the patients. SHS has become one of the most common complications after stroke according to the statistics (4). Although medical technology has made great progress, the pathogenesis of post-stroke SHS still remains unclear. Currently the theory of reflex sympathetic nerve damage holds the opinion that the occurrence of SHS is relevant to the autoimmune-mediated inflammatory (5) factors, but the opinion has not been proved by clinical trials. Therefore, effective means for the clinical treatment of SHS are indeficient at present, and the treatment taking the cause, pathogenesis and pathophysiology into consideration has been the important direction for rehabilitation treatment of post-stroke SHS. It was found through clinical trials that acupuncture in combination with routine rehabilitation therapy could relieve early pain, enhance QoL and improve social functions effectively for patients with post-stroke SHS.

\section{Patients and methods}

General data. A total of 178 patients with post-stroke SHS that received treatment in the Second Hospital of Dalian Medical University from March, 2012 to March, 2016 were selected into the study. They were divided into experimental group and 
control group (89 patients in each group). Patients in the control group included 53 males and 36 females aged 46-69 years (average, $53.35 \pm 3.30$ years); the course of the disease was 12 days to 3 months (average, $42.03 \pm 7.38$ days). Patients in the experimental group included 54 males and 35 females aged 47-70 years (average, $4.25 \pm 3.15$ years); the course of the disease was 11 days to 2.5 months (average, $41.43 \pm 8.01$ days). The comparisons of general data of clinical characteristics between the two groups are given in Table I.

Diagnostic criteria. i) Diagnosis of stroke: International diagnostic criteria for neurological cerebrovascular diseases (2015) (6) were taken for reference. ii) Diagnosis of SHS: Criteria in rehabilitation of post-stroke SHS (7) were taken for reference.

Diagnostic criteria: patients with cerebrovascular diseases in the past; patients suffering from pain in the shoulder and the hand and swelling of the shoulder, hand and fingers with or without limited mobility on the affected side for at least 3 months; patients that were free from trauma, infection and peripheral vascular diseases in the recent past.

The study was approved by the Ethics Committee of the Second Hospital of Dalian Medical University (Dalian, China).

Inclusion criteria. i) Patients that were diagnosed with stroke (phase I) through computed tomography (CT), magnetic resonance imaging (MRI) and clinical manifestations. ii) Patients who were aged 45-70 years. iii) Patients with the course of the disease ranging from 7 days to 3 months. iv) Patients that were informed, and agreed and signed the informed consent form.

Exclusion criteria. i) Patients with disturbance of consciousness such as somnolence and coma. ii) Patients with SHS caused by trauma and fracture. iii) Patients with transient ischemic attack. iv) Patients that were diagnosed with orthopedic disorders such as fracture of the upper extremity, scapulohumeral periarthritis and peripheral nerve injury, or mental diseases in the past. v) Patients with severe diseases of heart, kidney, liver or other organs. vi) Patients that failed to cooperate with examinations due to aphasia, loss of reading and dementia.

Treatment methods. Control group: Methods of rehabilitation training: i) Postural therapy: Lying on the uninjured side: The shoulder joint was stretched fully and bent at 90 degrees as much as possible under tolerable conditions; then the joints of the elbow, wrist and fingers were stretched respectively. Lying on the affected side: The shoulder joints were fully stretched forward under tolerable conditions; then joints of the elbow, dorsal extension wrist and fingers were stretched respectively. Supine position: Shoulder blades were stretched forward as much as possible under the circumstance that pain or distending pain was tolerable; then upper extremity, joints of the elbow, wrist and fingers were stretched. A bolster could be placed under the shoulder blade on the affected side to help patients recover. Sitting position: The affected extremity was placed on the shelf in front of the chest to make the shoulder blades maintain extended forward, thus preventing prolapse of the affected extremity. ii) Passive movement: Passive movement was conducted for joints of the shoulder, elbow, wrist and fingers of the affected extremity. Attention was paid to venous return when joints moved. The affected extremity was elevated appropriately to avoid congestion. iii) Active movement: Two palms of the hands touched each other with fingers interlocked. The elbow joint on the affected side was stretched actively. The uninjured upper extremity could appropriately assist the affected side to complete active movement of shoulder joint in the early stage, and the affected side was encouraged to move actively as much as possible in the late stage after the function of the patient was improved. The treatment duration was one month (45 min/time; once/day).

Experimental group: In addition to the rehabilitation training identical with that conducted for the patients in the control group, acupuncture was also carried out. The main acupoints were three Yang meridians and other meridians on the affected extremity [such as Jianyu (LI15), Jianliao (SJ14), Jianzhen (SI9), Jianneiling (EX-UE), Quchi (LI11), Shousanli (LI10), Hegu (LI4) and Waiguan (SJ5) on the affected side]. Acupuncture was conducted once per day for one month continuously and the needle-retaining time was 30 min each time.

Observation indexes. The following indexes of therapeutic efficacy were evaluated and recorded for all the cases in the experimental group and those in the control group before treatment and after one-month treatment: i) Assessment of motor function: Fugl-Meyer assessment (FMA) scale of the upper extremity was adopted (8). ii) Assessment of pain: Visual analogue scale (VAS) was adopted (9). iii) Assessment of comprehensive functions: Functional comprehensive assessment (FCA) was adopted (10). iv) Assessment of QoL: QoL assessment scale was adopted.

Evaluation of therapeutic efficacy. Improvement value $=$ the absolute value of (scores after treatment-scores before treatment). Improvement ratio $=$ improvement value/scores before treatment $\mathrm{x} 100 \%$. Improvement ratio $\geq 90 \%$ indicated cured; improvement ratio within $60-89 \%$ indicated improved; improvement ratio within 20-59\% indicated effective; improvement ratio $<20 \%$ indicated ineffective. Total effective rate $=[$ (clinical cured cases + improved cases + effective cases)/total cases] $\times 100 \%$.

Statistical analysis. Data treatment was conducted by the statistical software SPSS 21.0 (IBM, Armonk, NY, USA). $\chi^{2}$ test was adopted for the comparison of enumeration data, and t-test was used to compare measurement data. They were expressed as mean $\pm \mathrm{SD}$. $\mathrm{P}<0.05$ indicated that the differences had statistical significance.

\section{Results}

Comparisons of efficacy of early pain and improvement of upper extremity motor function between the two groups. The total effective rate of the improvement of early pain was $92.13 \%$ in experimental group and $68.53 \%$ in control group after onemonth treatment. The difference in the comparison between the two groups showed statistical significance $(\mathrm{P}<0.05)$. The 
Table I. Comparisons of general data between the two groups.

\begin{tabular}{lccc}
\hline General data & Experimental group & Control group & P-value \\
\hline Age (years) & $54.25 \pm 3.15$ & $53.35 \pm 3.30$ & $0.813^{\mathrm{b}}$ \\
Sex (male/female) & $54 / 35$ & $53 / 36$ & $0.878^{\mathrm{a}}$ \\
Course of the disease (day) & $41.43 \pm 8.01$ & $42.03 \pm 7.38$ & $0.752^{\mathrm{b}}$ \\
Affected extremity (left/right) & $51 / 38$ & $49 / 40$ & $0.763^{\mathrm{a}}$ \\
Scores of VAS & $6.59 \pm 1.98$ & $6.31 \pm 2.01$ & $0.756^{\mathrm{b}}$ \\
Scores of FMA & $25.03 \pm 7.37$ & $24.11 \pm 6.98$ & $0.841^{\mathrm{b}}$ \\
Scores of FCA & $27.08 \pm 6.68$ & $27.89 \pm 7.15$ & $0.755^{\mathrm{b}}$ \\
Scores of QoL & $117.28 \pm 27.03$ & $119.37 \pm 28.68$ & $0.798^{\mathrm{b}}$ \\
\hline
\end{tabular}

${ }^{a}$ Refers to $\chi^{2}$ test, $\mathrm{P}>0.05$; ${ }^{\mathrm{b}}$ refers to t-test, $\mathrm{P}>0.05$. The general data on clinical characteristics of patients in the two groups have no statistical difference. VAS, visual analogue scale; FMA, Fugl-Meyer assessment; FCA, functional comprehensive assessment; QoL, quality of life.

Table II. Comparisons of early pain and upper extremity motor function after treatment.

\begin{tabular}{|c|c|c|c|c|c|c|c|c|}
\hline Symptoms & Group & $\mathrm{n}$ & Cured & Improved & Effective & Ineffective & Total effective rate & P-value \\
\hline \multirow[t]{2}{*}{ Early pain } & Experimental group & 89 & 25 & 30 & 27 & 7 & $92.13 \%$ & \multirow[t]{2}{*}{$\mathrm{P}=0.01^{\mathrm{a}}$} \\
\hline & Control group & 89 & 11 & 19 & 31 & 28 & $68.53 \%$ & \\
\hline \multirow{2}{*}{$\begin{array}{l}\text { Upper extremity } \\
\text { motor function }\end{array}$} & Experimental group & 89 & 13 & 25 & 35 & 16 & $82.02 \%$ & \multirow[t]{2}{*}{$\mathrm{P}=0.05^{\mathrm{b}}$} \\
\hline & Control group & 89 & 6 & 19 & 34 & 30 & $66.29 \%$ & \\
\hline
\end{tabular}

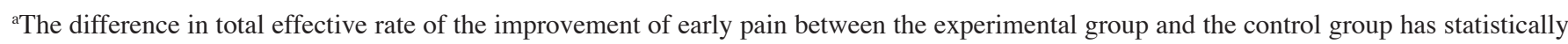
significance $\left(\chi^{2}=12.68, \mathrm{P}=0.01<0.05\right)$; ${ }^{\mathrm{b}}$ The difference in the improvement of upper extremity motor function between the experimental group and the control group has statistical significance $\left(\chi^{2}=5.746, \mathrm{P}<0.05\right)$.

experimental group $(82.02 \%)$ had better effect than the control group $(66.29 \%)$ in the improvement of upper extremity motor function, and the difference showed statistical significance $\left(\chi^{2}=5.746, \mathrm{P}<0.05\right)($ Table II).

Comparisons of each index between the two groups after treatment. The differences in the comparisons of FMA scores of motor function, VAS scores, FCA scores and QoL scores before treatment had no statistical significance $(\mathrm{P}>0.05)$. They were comparable (Table I). The values of each assessment index obtained after one-month treatment were compared with those obtained before treatment, which showed that both the experimental group and the control group had statistically significant differences $(\mathrm{P}<0.05)$. All the assessment indexes had some improvements after one-month treatment compared with those before treatment (Table III). However, the improvement values of each index in the experimental group were better than those in the control group. The differences had statistical significance $(\mathrm{P}<0.05)$ (Table IV).

\section{Discussion}

SHS is a common complication after stroke. If it is not treated timely, contracture and deformity of joint muscles may be caused finally, thus resulting in permanent disability, which will bring great pain to the patients. Therefore, it is of great importance to carry out early diagnosis and early inference for patients in stage I. Active and effective treatment can reduce or avoid occurrence or development of the disease to stages II and III (11). The ATLANTIS study (12) indicated that functional recovery in stroke patients was associated with the starting time of early rehabilitation therapy. Therefore, it is recommended to carry out treatment as early as possible after the disease is stabilized. There are many methods for the treatment of poststroke SHS currently, including anti-inflammatory, analgesic and nerve-nourishing treatments with Western medicines, and acupuncture, massage, rehabilitation therapy and physical therapy with traditional Chinese medicines, but the efficacy needs to be improved. The efficacy of acupuncture therapy, a form of the treatment with traditional Chinese medicines, in the treatment of post-stroke diseases has been clinically affirmed (13-15). However, there are few studies on the use of acupuncture therapy in the treatment of pain for patients with post-stroke SHS in China or other countries, especially the studies on early pain recovery of post-stroke SHS. Therefore, traditional acupuncture therapy in combination with rehabilitation therapy was compared with pure rehabilitation therapy in this study to explore the efficacy of acupuncture in the treatment of pain or recovery in patients with post-stroke SHS, thus seeking an effective treatment method.

The main theoretical mechanism for acupuncture to relieve pain has not yet been studied. Some scholars agree with gate-control theory put forward by Melzack et al (16), while others with Chen et al (17) who proposed another point 
Table III. Comparisons of assessment indexes between the two groups after treatment and comparisons of assessment indexes obtained before treatment with those obtained after treatment in each group.

\begin{tabular}{|c|c|c|c|c|c|c|}
\hline \multirow[b]{2}{*}{$\begin{array}{l}\text { Assessment } \\
\text { indexes }\end{array}$} & \multicolumn{2}{|c|}{ After treatment } & \multirow[b]{2}{*}{ P-value ${ }^{a}$} & \multicolumn{2}{|c|}{ Before treatment } & \multirow[b]{2}{*}{ P-value ${ }^{b}$} \\
\hline & $\begin{array}{l}\text { Experimental } \\
\text { group }\end{array}$ & $\begin{array}{l}\text { Control } \\
\text { group }\end{array}$ & & $\begin{array}{l}\text { Experimental } \\
\text { group }\end{array}$ & $\begin{array}{l}\text { Control } \\
\text { group }\end{array}$ & \\
\hline VAS & $2.53 \pm 1.78$ & $3.35 \pm 2.07$ & 0.01 & $6.59 \pm 1.98$ & $6.31 \pm 2.01$ & $0.01 / 0.02$ \\
\hline FMA & $39.98 \pm 8.72$ & $32.02 \pm 7.29$ & 0.008 & $25.03 \pm 7.37$ & $24.11 \pm 6.98$ & $0.00 / 0.01$ \\
\hline FCA & $52.23 \pm 14.36$ & $42.01 \pm 19.28$ & 0.003 & $27.08 \pm 6.68$ & $27.89 \pm 7.15$ & $0.00 / 0.02$ \\
\hline QoL & $212.13 \pm 27.18$ & $193.67 \pm 27.01$ & $>0.05$ & $117.28 \pm 27.03$ & $119.37 \pm 28.68$ & $0.02 / 0.03$ \\
\hline
\end{tabular}

${ }^{a}$ The comparisons of scores of VAS, FMA, FCA and QoL between the experimental group and the control group after one-month treatment have statistical difference $(\mathrm{P}<0.05)$. ${ }^{\mathrm{b}} \mathrm{P}-\mathrm{v}$ alues of the comparisons of scores of VAS, FMA, FCA and QoL obtained before treatment and those obtained after treatment in each group are $<0.05$. Symptoms of the patients in the two groups are improved, and there are statistical differences in the comparisons of the values obtained after treatment with those obtained before treatment $(\mathrm{P}<0.05)$. VAS, visual analogue scale; FMA, Fugl-Meyer assessment; FCA, functional comprehensive assessment; QoL, quality of life.

Table IV. Comparisons of improvement values of scores of VAS, FMA, FCA and QoL between the two groups of patients with SHS after treatment.

\begin{tabular}{lccc}
\hline $\begin{array}{l}\text { Assessment } \\
\text { indexes }\end{array}$ & $\begin{array}{c}\text { Experimental } \\
\text { group }\end{array}$ & $\begin{array}{c}\text { Control } \\
\text { group }\end{array}$ & P-value \\
\hline VAS & $3.98 \pm 0.86$ & $3.53 \pm 0.64$ & $0.001^{\mathrm{a}}$ \\
FMA & $14.45 \pm 3.31$ & $8.73 \pm 3.03$ & $>0.05$ \\
FCA & $26.37 \pm 5.64$ & $17.66 \pm 3.45$ & $0.002^{\mathrm{a}}$ \\
QoL & $100.51 \pm 13.84$ & $76.68 \pm 12.46$ & $>0.05$ \\
\hline
\end{tabular}

${ }^{\mathrm{a}} \mathrm{P}<0.05$ indicates that the improvement conditions of patients with SHS in the experimental group are better than those in the control group after treatment. SHS, shoulder-hand syndrome; VAS, visual analogue scale; FMA, Fugl-Meyer assessment; FCA, functional comprehensive assessment; QoL, quality of life.

of view (the theory of the release of endogenous analgesic substances). Human body's analgesic system can release and secrete endogenous substances with analgesic effects (such as $\mathrm{M}$ receptors and opioids) to improve human body's tolerance to pain, and it can also inhibit the role of pain-causing transmitters, including adrenaline, so as to achieve analgesic effect $(4,18,19)$. Acupuncture can make the analgesic system work by stimulating nervous system of the body to achieve analgesic effect. The results of this study showed that after one-month treatment, the total effective rate of early pain was $92.13 \%$ for patients with post-stroke SHS in the experimental group and $68.53 \%$ for those in the control group receiving rehabilitation therapy alone. The efficacy of the experimental group was significantly better than that (70.1\%) of the study conducted by O'Connell et al (20) and that of the study conducted by Lee et al (21) in which glucocorticoids were used to treat post-stroke SHS. The results also indirectly confirmed the efficacy of acupuncture therapy in the treatment of post-stroke SHS. The difference in the effective rate of the efficacy may be relevant to the selection of cases and physicians. In this study, the patients were selected strictly as per the diagnosis of stroke and SHS. In addition, acupuncture therapies for all the patients in the experimental group were conducted by physicians with the title of associate chief physician that have engaged in clinical acupuncture for a long time strictly as per standards to reduce differences in treatment as much as possible.

Stroke is treated to reduce the occurrence of post-stroke complications as much as possible on the basis of treating the primary disease so as to improve QoL. Results of this study indicated that $82.02 \%$ of the patients with post-stroke SHS in the experimental group showed improvement in the function of upper extremity after one-month treatment with acupuncture in combination with rehabilitation therapy, while the total effective rate of the patients in the control group receiving single rehabilitation therapy was only $66.29 \%$. The efficacy of acupuncture in combination with rehabilitation therapy was better than that of rehabilitation therapy, and its results were basically identical with those of the study conducted by Sun et al (22). Melzack et al (16) showed that blood flow volume, edema and pain of the affected extremity were improved and QoL was also enhanced greatly after the patients with post-stroke SHS received acupuncture therapy. The results of this study revealed that patients in the two groups had some improvements in QoL, but the efficacy of the experimental group was better than that of the control group, which further affirmed the results of the study conducted by Melzack et al (16).

In conclusion, early pain and motor function of the affected extremity and QoL can be improved obviously after the patients with post-stroke SHS receive acupuncture in combination with rehabilitation therapy. Acupuncture plus rehabilitation training are worthy to be popularized clinically.

\section{References}

1. Hannan MA, Sabeka MM and Miah BA: Shoulder hand syndrome in hemispheric stroke. J Neurol Sci 3: 167-173, 2013.

2. Kondo I, Hosokawa K, Soma M, Iwata M and Maltais D: Protocol to prevent shoulder-hand syndrome after stroke. Arch Phys Med Rehabil 82: 1619-1623, 2001. 
3. Jun W, Xiao C, Jian P, Huanhuan N, Cuixia Z, Chuishui H, Mei H, Peiyu Q and Ying D: Timeliness of the analgesic effect of superficial needling on shoulder-hand syndrome after stroke. World J Acupunct Moxibust 4: 5-10, 2015.

4. Li N, Tian F, Wang C, Yu P, Zhou X, Wen Q, Qiao X and Huang L: Therapeutic effect of acupuncture and massage for shoulderhand syndrome in hemiplegia patients: A clinical two-center randomized controlled trial. J Tradit Chin Med 32: 343-349, 2012.

5. Borchers AT and Gershwin ME: Complex regional pain syndrome: A comprehensive and critical review. Autoimmun Rev 13: 242-265, 2014.

6. Offner H, Ihara M, Schäbitz WR and Wong PT: Stroke and other cerebrovascular diseases. Neurochem Int 107: 1-3, 2017.

7. Cheng YY, Shu JH, Hsu HC, Liang Y, Chang ST, Kao CL and Leu HB: The impact of rehabilitation frequencies in the first year after stroke on the risk of recurrent stroke and mortality. J Stroke Cerebrovasc Dis 6: 1052-1057, 2017

8. Woytowicz EJ, Rietschel JC, Goodman RN, Conroy SS, Sorkin JD, Whitall J and McCombe Waller S: Determining levels of upper extremity movement impairment by applying a cluster analysis to the Fugl-Meyer assessment of the upper extremity in chronic stroke. Arch Phys Med Rehabil 98: 456-462, 2017.

9. Behrend CJ, Schönbach EM, Vaccaro AR, Coyne E, Prasarn ML and Rechtine GR: Maximum pain on visual analog scales in spinal disorders. Spine J 17: 1061-1065, 2017.

10. Turk DC, Fillingim RB, Ohrbach R and Patel KV: Assessment of psychosocial and functional impact of chronic pain. J Pain 17 (Suppl 9): T21-T49, 2016.

11. Berglund A, Schenck-Gustafsson K and von Euler M: Sex differences in the presentation of stroke. Maturitas 99: 47-50, 2017.

12. Hacke W, Donnan G, Fieschi C, Kaste M, von Kummer R, Broderick JP, Brott T, Frankel M, Grotta JC, Haley EC Jr, et al; NINDS rt-PA Study Group Investigators: Association of outcome with early stroke treatment: Pooled analysis of ATLANTIS, ECASS, and NINDS rt-PA stroke trials. Lancet 363: 768-774, 2004

13. Yongfeng L, Xiaoming M, Bing Y, Yanhua G, Shaoyun Z and Xiaodan R: Warm needling on the four knee acupoints combined with Chinese herb in the treatment of knee pain after stroke. World J Acupunct Moxibust 25: 21-25, 2015.
14. Wong IS, Ng KF and Tsang HW: Acupuncture for dysphagia following stroke: A systematic review. Eur J Integr Med 4: e141-e150, 2012.

15. Yixu H, Yanjun W, Yebo W and Limin C: Essence of GAO Yu-chun's experience in treatment of stroke. World J Acupunct Moxibust 26: 42-48, 2016.

16. Melzack R, Stillwell DM and Fox EJ: Trigger points andacupuncture points for pain: Correlations and implications. Pain 3: 3-23,1977.

17. Chen L, Zhang J, Li F, Qiu Y, Wang L, Li YH, Shi J, Pan HL and Li M: Endogenous anandamide and cannabinoid receptor-2 contribute to electroacupuncture analgesia in rats. J Pain 10: 732-739, 2009.

18. Shang Y, Ma CC, Cai Y, Wang DS and Kong LL: Clinical study on acupuncture combined with rehabilitation therapy for treatment of post-stroke shoulder-hand syndrome. Zhongguo Zhen Jiu 28: 331-333, 2008 (In Chinese).

19. Li W, Kang X, Ren JL, Lai XZ and Tai LW: Effects of extended in-patient treatment training on outcome of post-stroke dysphagia. Eur Rev Med Pharmacol Sci: ERMPS-12713 (Epub ahead of print)

20. O'Connell NE, Wand BM, McAuley J, Marston L and Moseley GL: Interventions for treating pain and disability in adults with complex regional pain syndrome. Cochrane Database Syst Rev 4: CD009416, 2013.

21. Lee SK, Yang DS, Lee JW and Choy WS: Four treatment strategies for complex regional pain syndrome type 1 . Orthopedics 35: e834-e842, 2012.

22. Sun Y, Wang Y and Wang W: Effect of acupuncture plus rehabilitation training on shoulder-hand syndrome due to ischemic stroke. J Acupunct Tuina Sci 10: 109-113, 2012. International (CC BY-NC-ND 4.0) License. 